\title{
Das Geschlecht der Dinge. Interdisziplinäre und epochenübergreifende Perspektiven auf Geschlecht, Lebensstil und den Symbolcharakter der Dinge.
}

Eine Tagung des SFB 496 "Symbolische Kommunikation und gesellschaftliche Wertesysteme vom Mittelalter bis zur Französischen Revolution « und des Arbeitskreises Historische Frauen- und Geschlechterforschung (AKHFG) vom 26. bis 28. Juni 2003 in Münster.

Dem aufklärerischen Denken galten Dinge einfach nur als Dinge, zu Nutzen und Gebrauch des Menschen. Diese Annahme vom bloßen Vorhandensein der Gegenstände verstellte lange Zeit den Blick für die Macht der Dinge und die Macht über Dinge, die zu allen Zeiten die Gestalt der Gesellschaft mit bestimmten. Neuere kulturwissenschaftliche Studien belegen: Ein soziales Gefüge ist stets geprägt durch die von ihm erzeugten und genutzten Gegenstände. In Dinge wird die Ordnung der Gesellschaft eingeschrieben - durch Besitzstrukturen, Verwendungspraktiken und Bedeutungsbelegungen. Die Frage nach Objekten gehört bislang zu den unterbelichteten Randzonen der Sozial- und Kulturwissenschaften. Noch seltener hat man in diesem Zusammenhang die Bedeutung geschlechtlicher Zuschreibungen hervorgehoben.

In den einführenden Statements zur Tagung betonten daher Barbara Stollberg-Rilinger für den SFB 496 sowie Martina Kessel, Wiebke Kolbe und Gabriela Signori für den AKHFG die Zielsetzung, einen Überblick über gegenwärtige Tendenzen der Forschung durch konkrete Projekte zu bieten. Auf diese Weise sollten Anstöße für ein systematisches Nachdenken über das "Geschlecht der Dinge " gegeben werden. In fünf Sektionen debattierten etwa hundert Teilnehmerinnen und Teilnehmer an drei Ta- gen über Dinge, die vererbt, am Körper getragen und als Kulturgüter begriffen werden, die eine Bedeutung für die Essund Trinkkultur haben und die erinnert werden. In den zwanzig Beiträgen aus den Disziplinen Archäologie, Geschichtswissenschaft, Volkskunde, Ethnologie und Textilwissenschaft wurde überzeugend aufgezeigt, dass das Geschlecht einer Sache kaum jemals sächlich ist.

Mehrere Beitragende argumentierten aus einer akteursorientierten Perspektive und fragten nach Handlungsspielräumen unter der Bedingung rechtlich festgeschriebener Besitzstrukturen. Eva-Maria Butz (Mediävistik/Dortmund) und Volker Lünnemann (Neuere Geschichte/ Münster) zeigten, dass rechtlich scheinbar klare Erbregelungen verschiedentlich unterlaufen wurden. Anhand von Streitfällen aus dem 13. Jahrhundert wies Eva-Maria Butz nach, dass die Witwen eines Dynasten keineswegs immer ihrer Bestimmung folgten, sich durch die Nutzung einer "Wittumsburg“ zu ihren Lebzeiten versorgen zu lassen. Verzichtete die eine aus religiösen Motiven auf ihr Verfuigungsrecht, was die Erben um ihren Besitz fürchten ließ, so verlangte die andere mehr als ihr rechtlich zustand und verfuhr mit der Burg wie mit ihrem Eigengut. Volker Lünnemann wiederum setzte das Anerbenrecht des 19. Jahrhunderts auf den Prüfstand, mit dem das Vererben eines 
bäuerlichen Hofes in männlicher Linie gesichert schien. Unter bestimmten Voraussetzungen der Familienkonstellation hatten jedoch auch Frauen gute Chancen, den Hof zu erben. Die scheinbare Eindeutigkeit vergeschlechteter Vererbungsstrukturen wird mit der Betrachtung der Strategien der historischen Akteure hinterfragbar. Der Besitz und Gebrauch anderer Dinge war nicht per Sachenrecht geregelt, sondern durch symbolische $\mathrm{Zu}$ ordnungen bestimmt. Ein vermeintlich geschlechtsloses Ding konnte für einen anderen Komplex stehen, so das Buch für die Bildung. Antje Flüchter-Sheryari (Frühneuzeitliche Geschichte/Münster) demonstrierte, wie im Verlauf des 18. Jahrhunderts das Verhältnis von Frauen zum Buch als gefährdend für "ihre Weiblichkeit" beschrieben und damit ihr Ausschluss von der Gelehrsamkeit normativ festgeschrieben wurde. Flüchters Akteurin, die französische Schriftstellerin Sophie La Roche, bemühte sich jedoch, durch geschicktes Umformen der Vorstellungen der bürgerlichen Meisterdenker die zulässigen Grenzen zu verschieben.

Die Frage nach Praxisformen, nach Handlungsweisen im Umgang mit Dingen stand im Vordergrund weiterer Beiträge. Elke Hartmann (Alte Geschichte/Berlin) betrachtete mit der Toga ein Kleidungsstück im Athen des 6. bis 4. Jahrhunderts v. Chr. und wies nach, dass die gesellschaftliche Norm zwar eine soziale Differenzierung anhand der Kleidung verbot, trotzdem durch die Art des Tragens der Einheitskleidung Unterschiede markiert wurden. Die gekonnte Bändigung des Stoffs kennzeichnete den männlichen freien Bürger in politischer Führungsposition. Gleich zwei Referate stellten den Trinkkrug in den Mittelpunkt. Jan Rüdiger (Mediävistik/Berlin) beschrieb ein gängiges literarisches $\mathbf{E r}$ zählmotiv aus dem hochmittelalterlichen Skandinavien, das eine - in seiner Abfolge genau definierte - Praxis als für die Zeitgenossen praktikabel ausweist. In prekären Situationen, in denen ein Fremder eintraf, konnte ein Mädchen diesem durch das Darreichen eines Trinkbechers das Gasttum anbieten und eine Konfrontation der Männer vermeiden. Bei Sabine von Heusinger (Mediävistik/Mannheim) symbolisierte das gemeinsame Trinken im Männerbund der Gesellenvereine den Zusammenhalt einer neuen sozialen Gruppierung, die sich Ende des 14. Jahrhunderts formierte. Ähnlich argumentierte Barbara Krug-Richter (Volkskunde/Münster) in ihrem Vortrag "Von Messern und Männlichkeit «, in dem sie den öffentlichen provokanten Umgang von Studenten mit ihren Waffen als Abgrenzung männlicher Gruppen untereinander deutete. Im Gebrauch von Dingen manifestiert sich somit eine soziale Beziehung der Beteiligten. Der Umgang mit Objekten folgt bestimmten Handlungsschemata, die kollektiv von einer Gruppe ausgeübt und als sinnvolle Praxis wahrgenommen werden. Auch wenn dies nicht immer explizit angemerkt wurde, stand hier die Habitustheorie Pierre Bourdieus Pate.

Manche Dinge erfüllen im Zusammenhang mit der analytischen Kategorie Geschlecht geradezu eine Stellvertreterfunktion, so eng sind sie mit Vorstellungen von Weiblichkeit oder Männlichkeit verbunden. Mehrere Beiträge der Tagung widmeten sich ausgesprochen 'weiblichen Dingen. Die mittelalterliche "Gerade" stellte Karin Gottschalk (Frühneuzeitliche Geschichte/Kassel) vor. Unter dieser Bezeichnung fasste das mittelalterliche Sachenrecht einen Katalog von Dingen, die nur der Hausmutter als vererbbarer Besitz zustanden - Hausrat, Textilien und Arbeitsgeräte - und dem Haushalten und Kindergebären zugeordnet waren. Eine neuzeitliche Entsprechung fand sich in den elektrifizierten Küchengeräten, die in den 1950er Jahren in die bundesdeut- 
schen Haushalte Einzug hielten. Ulrike Lindner (Neuere Geschichte/München) zeigte, dass in dieser Epoche die geschlechtsspezifische Zuordnung der Dinge weniger über das Sachenrecht, sondern über die bürgerliche Geschlechterideologie und ihre moderne Arbeitsteilung geregelt wurde. Die Haushaltsgeräte stabilisierten diese Vorstellung, indem plausibel gemacht wurde, dass mit ihrer Hilfe die gesamte Haushaltsarbeit von einer (weiblichen) Person zu bewältigen war. Tatsächlich belegen Zeitstudien jedoch, dass durch die Technisierung kaum Arbeitszeit eingespart werden konnte. Hand und Messer blieben die wichtigsten Küchengeräte. Christine Kunst (Alte Geschichte/ Potsdam) sprach über die Ornamenta uxoria, den Schmuck der römischen Ehefrau. Kunst konnte zeigen, dass die Ornamenta aufgrund ihrer variantenreichen Funktionalität einer starken Standardisierung unterworfen waren. Sie dokumentierten Statusunterschiede, legten die Frauen auf die körperliche Sphäre fest und erinnerten Matronen der Oberschicht an die Pflicht der Geburt. Einen Sonderfall der Repräsentation von Weiblichkeit stellte Annemarie Fiedermutz (Ethnologie/Münster) in ihrem Vortrag über anthropomorphe Formen des Bauens bei einer westafrikanischen Ethnie im späten 20. Jahrhundert vor. Bei den Kasena wird die in der Kultur zentrale weibliche Funktion - die Fruchtbarkeit - permanent in Architektur und Inneneinrichtung ausgedrückt. Die dem weiblichen Körper nachgestaltete Form der Lehmbauten und der Einrichtungsgegenstände markiert, ständig im alltäglichen Lebensumfeld präsent, das Geschlecht und seine Aufgaben.

In vielen Beiträgen der Tagung stand die Frage nach der Biografie der Dinge im Mittelpunkt, nach Wandel und Kontinuität in den geschlechteten Bedeutungszuschreibungen. Karin Hausen (Neueste Geschichte/Berlin) sah in dem Beginn des Zeitalters des Massenkonsums eine signifikante Zäsur für die allgemeine Betrachtung der Dinge. In der Konsumgesellschaft sind nicht mehr Sparsamkeit, Haltbarkeit und pfleglicher Umgang mit den Dingen handlungsleitend, sondern die gekonnte Handhabung des Überflusses. In Hausens öffentlich gehaltenem Festvortrag ging es um den neuartigen Warenkult, der in starkem Ausmaß durch die Werbung getragen wird. In der industriellen Massenkultur verloren die Dinge spätestens seit den 1980er Jahren angesichts des immer schnelleren Wechsels der Warenpalette ihre stabile Aussagekraft und sind gegenwärtig, so Karin Hausen, nur noch kurzfristig mit einem Geschlecht verknüpfbar. Eine weitere Zäsur für das Umgehen mit Dingen setzte Karin Gottschalk bereits im 18. Jahrhundert, indem sie am Beispiel der Gerade das gewandelte Verständnis im Sachenrecht darlegte. Während zuvor die Dinge nach der ständischen Vorstellung "Jedem das Seine" zugewiesen wurden, büßten sie seit dem 18. Jahrhundert ihre explizite Geschlechtlichkeit weitgehend ein. Der Frauenbesitz erschien nun als ungerechtfertigtes Privileg, das dem Prinzip der Allgemeinheit des Rechts zuwiderlief. Gabriela Signori (Mediävistik/Münster) legte in ihrem Vortrag über "Schleier, Hut oder Haare" die Biografie dieser Dinge seit dem 4. bis in das 14. Jahrhundert hinein dar. Die Frage, wie, wann und von wem das Haupt jeweils zu bedecken war, erwies sich als konstitutiv für die religiöse Ordnung. Sie diente als Mittel der Unterscheidung zwischen Frauen und Männern, Geistlichen und Laien, höheren und niederen Ständen.

Allerdings wies Gabriela Signori vor dem Hintergrund ihres Themas auch auf die Stabilität der Bedeutung von Gegenständen hin, die in manchen Fällen über mehrere Epochen hinweg reichen kann. Die Wirkungsgeschichte des Korinther- 
briefes des Paulus setzt sich bis in die Gegenwart fort, denn in einigen Orten ist es nach wie vor üblich, dass Frauen ihren Kopf während des Gottesdienstes bedecken. In die gleiche Richtung argumentierte auch Simona Slanicka (Mediävistik/Bielefeld) in ihrem Referat über "Das Geschlecht der Haare", in dem die kulturelle Bedeutung von Haaren in biblischen Passagen und Heiligenviten thematisiert wurde. Slanicka hob die auffallende Kontinuität des Scherens von Frauenhaaren als Sanktionsmittel hervor, die bis in das 20. Jahrhundert hinein reicht. In einem interkulturellen Vergleich ermittelte Natascha Sojc (Klassische Archäologie/München) Kontinuitäten im Prozess des Trauerns um Verstorbene von der Antike bis zur Gegenwart. Bilddarstellungen auf Grabreliefs der attischen Demokratie des 5. und 4. Jahrhunderts v. Chr. stellte die Referentin Fotografien aus dem Jahr 1999 gegenüber, mit denen die Trauer um eine Verstorbene veröffentlichtkünstlerisch umgesetzt wurde. Sojc erkannte in beiden Kulturen eine bildhafte Idealisierung der Toten als tröstlichen Rückbezug für die Lebenden. Der Beitrag von Claudia Lenz (Neuere Geschichte/Hamburg) behandelte eine zentrale Voraussetzung für die Stabilität von Dingbedeutungen: die Erinnerung. Unter Bezug auf Aleida Assmanns Überlegungen zum kulturellen Gedächtnis analysierte Lenz die kollektive Erinnerung an den norwegischen Widerstand gegen die deutsche Besatzung im Zweiten Weltkrieg. Als Erinnerungsobjekte stellte sie Fotografien in den Mittelpunkt. Durch die Auswahl der Zeitgenossen und Nachlebenden wird die geschlechtsspezifische Arbeitsteilung 'privat-öffentlich' auch in den Blick zurück eingeschrieben.

Die Sexualisierung von Objekten wurde in zahlreichen Beiträgen thematisiert. Dabei herrschte weitgehende Übereinkunft, dass die Dinge vor allem dann eine sexuelle Komponente erhalten, wenn sie in Beziehung zu Konzepten von ,Weiblichkeit stehen. Frauenhaar und Kopfbedeckung waren laut Simona Slanicka und Gabriale Signori im Mittelalter eng mit der Sexualität der Trägerin verknüpft, während bei den Männern eher Eigenschaften wie Kühnheit oder Weisheit, Rang und Stand angezeigt wurden. Stefan Haas (Neuere Geschichte/Münster) stellte in seiner "Verr(a)uchten $\mathrm{Ge}$ schichte" dar, dass rauchende Frauen in den zwanziger Jahren eine Sexualisierung erfuhren. Der Rauch der Zigarette diente dazu, den Frauenkörper auf geheimnisvolle Weise $\mathrm{zu}$ verschleiern und zugleich zu erweitern. Elke Gaugele (Textilwissenschaft/Köln) behandelte in ihrem Vortrag den Prozess der symbolischen und performativen Verknüpfung von "Frau", "Schürze" und "Fetisch" seit dem ausgehenden 19. Jahrhundert. Von einem Symbol der Häuslichkeit entwickelte sich die Schürze zu einem sexualisierten Zeichen. Gaugele bewertete den Fetischismus im Sinne Michel Foucaults als eine MenschDing-Beziehung, die als Modellperversion zum Leitfaden der Deutung aller Abweichungen wurde. Gabriela Signori warb für den Versuch, in dieser Frage eine Periodisierung vorzunehmen und $\mathrm{zu}$ ermitteln, wann welche Dinge anfingen, die Funktion der Sexualisierung zu besetzen.

Das Verhältnis zwischen Mensch und Ding wurde auf der Tagung meist vom Menschen her gedacht. Es ging um das, was den Dingen geschieht - sie werden besessen, geformt, gebraucht, verbraucht und verpersönlicht. Ist der Mensch somit das $\mathrm{Ma}$ aller Dinge? Stefan Haas verwies mit dem flüchtigen Objekt "Rauch" auf den Eigencharakter von Dingen und auf Grenzen der Verfügungsmacht über sie. Die Einverleibung und Neugeburt der Objekte durch diskursive Konstruktionsprozesse könne wie der Zigarettenrauch, der den Mund verlässt, nicht beliebig in 
ihrem Weg gesteuert werden. Damit werde auch die These fragwürdig, dass sich Diskurse in Dinge einschreiben und wieder verändern könnten, gerade weil diese Dinge stabil seien. Bettina Mann (Ethnologie/Halle) betonte ebenfalls in ihrem Vortrag "Lang gekocht und schnell gegessen“ die Mobilität der Materialität von Objekten, die im Umgehen mit Nahrungsmitteln offensichtlich wird. Der Transformationsprozess der Produkte von roh über gekocht bis zu verdaut - sei als ein Aspekt des Essens bislang noch wenig beachtet worden. Simona Slanicka hob den von ihr vorgestellten Gegenstand, das Haar, als Grenzgänger unter den Dingen hervor. Es sei ein Objekt am Körper, das auf Repräsentationen des Körpers verweise. In der Diskussion über diese Beiträge forderte das Plenum, grundsätzlicher über das Verhältnis von Mensch und Objekt nachzudenken. Verwiesen wurde auf den französischen Wissenschaftssoziologen Bruno Latour, der rät, stärker von den Dingen selbst auszugehen und ihnen als "nichtmenschlichen Wesen « Respekt zu erweisen.

Faszinieren konnte die Variationsbreite der methodischen und theoretischen $\mathrm{Zu}$ griffsweisen im Blick auf die Dinge: aus der Perspektive der Akteure, durch symbolische Bedeutungssetzung in Diskursen und Praktiken, im interkulturellen, epochenübergreifenden Vergleich (Natascha Sojc) und mittels EDV-gestuitzter Erhebung von Massendaten (Volker Lünnemann). Die Ethnologinnen Annemarie Fiedermutz und Bettina Mann steuerten für die Analyse des Umgehens mit Dingen die teilnehmende Beobachtung bei. Bettina Mann stellte anhand von Daten, die sie im Rahmen einer Feldforschung in der Metropole Kairo in den 1990er Jahre erhoben hatte, die zentrale Funktion der Speisenauswahl und der Nahrungsversorgung für die häusliche Arbeitsteilung dar. Kulinarische Ordnungen erweisen sich als Bestandteil des kulturellen Alphabets. Marita Bombek (Textilwissenschaft/ Köln) schließlich zielte darauf, in der interdisziplinären Zugriffsweise ihres $\mathrm{Fa}$ ches ein bislang wenig beachtetes Objekt mit seinem besonderen Quellenwert zu nutzen. Mittelalterliche, von adeligen Frauen selbst hergestellte Bildteppiche sollen als Medien der Erzählung, der Erinnerung und der Repräsentation erkannt und erforscht werden.

Leicht hätte angesichts der Vielzahl an Themen und Herangehensweisen die Übersicht verloren gehen können. Die Organisatorinnen der Tagung setzten daher einen Abschlusskommentar an das Ende, den Katharina Simon-Muscheid (Mediävistik/Basel) übernahm. Sie fasste die thematischen Leitlinien der Beiträge, die sich auch in diesem Bericht spiegeln, treffsicher zusammen und strukturierte auf diese Weise die von ihr moderierte Abschlussdiskussion. Der nächste Schritt in der Forschung über die Dinge und ihr Geschlecht könnte in einem grundlegenden Austausch über theoretische Vorannahmen und Begriffsverwendungen liegen. Wie wird das Verhältnis von (vergeschlechtlichtem) Mensch, Ding, Materialität, Diskurs und Praxis definiert? Gefragt werden sollte auch, durch welche Prozesse es gesellschaftlichen Machtinstanzen gelingt, ihre jeweilige symbolische Bedeutungssetzung für Objekte akzeptabel und praktikabel zu machen. Dinge erweisen sich somit als Kristallisationspunkte geschlechterbestimmter $\mathrm{Zu}$ schreibungen. In ihnen fokussieren sich, gleichsam wie unter dem Brennglas, zentrale Probleme der Macht und Ohnmacht, Ordnung und Umordnung, von Kontinuität und Wandel in den Geschlechterverhältnissen. 